The feasibility of PAIC for the PRIMA and PAOLA-1 trials was assessed based on assumptions outlined in the guidance by the Decision Support Unit in NICE DSU Technical Support Document 18; PFS was the outcome for the analysis.

Results All 12 RCTs assessed for ITC feasibility were excluded based on various factors including: the lack of a common comparator with PRIMA within the network (ICON-7, GOG0218, PAOLA-1, VELIA/GOG-3005); differing measurement of PFS and overall survival starting timepoint due to trial design (ICON-7, GOG-0218, VELIA/GOG-3005); inclusion of stage III patients with no visible residual disease following debulking surgery (PAOLA-1, SOLO-1, VELIA/GOG-3005); disparity between disease biomarker (SOLO-1).

For the PAIC, three fundamental differences between the PRIMA and PAOLA trials were identified; inclusion criterion related to residual disease was wider in PAOLA meaning that the 'conditional constancy of absolute effects' was violated; receipt of neoadjuvant chemotherapy was identified as a confounding factor that would bias a PAIC; discrepancies in the assessment of type and frequency of measurement of the PFS outcome.

Conclusions Based on the evidence currently available, neither an ITC nor PAIC would meet current guidelines, such as those outlined by the International Society for Pharmacoeconomics and Outcomes Research, for these analyses. Their results would not be considered appropriate evidence for use in clinical decision making or reimbursement decisions. The extent of imbalance caused by differences in the patient inclusion/exclusion criteria for intended comparisons is unknown and a recognised limitation of PAICs.

Disclosures This study was funded by GlaxoSmithKline.

Due to lengthy author disclosures, author COI information will be provided directly to the congress.

\section{THE USE OF REAL-WORLD EVIDENCE FROM THE EDINBURGH OVARIAN CANCER DATABASE TO EXPLORE A DATA GAP IN THE PRIMA TRIAL}

${ }^{1}$ Robert L Hollis, ${ }^{2}$ Zsofia Kiss, ${ }^{2}$ Nichola Roebuck, ${ }^{2}$ Helen Starkie-Camejo, ${ }^{2}$ Kiera Heffernan, ${ }^{1}$ Charlie Gourley. ${ }^{1}$ Nicola Murray Centre for Ovarian Cancer Research, Cancer Research UK Edinburgh Centre, MRC Institute of Genetics and Molecular Medicine, Edinburgh, UK; ${ }^{2}$ GlaxoSmithKline Brentford, Middlesex, London, UK

10.1136/ijgc-2020-ESG0.126
Introduction/Background Niraparib is an oral, highly selective poly(ADP-ribose) polymerase inhibitor (PARPi). The PRIMA/ ENGOT-OV26/GOG-3012 trial intention-to-treat population included stage III and IV ovarian cancer (OC) patients postprimary or interval debulking surgery (PDS/IDS) irrespective of residual disease (visible residual disease [VRD] or no VRD [NVRD]), except for stage III patients post-PDS with NVRD. A data gap therefore exists in the stage III postPDS NVRD population. This study aims to use real-world evidence from the Edinburgh Ovarian Cancer Database to explore the difference in overall survival (OS) and progression-free survival (PFS) in: a) a 'simulated-PRIMA cohort' (stage III VRD after PDS, stage III and IDS, and stage IV OC), b) a 'simulated-NVRD after PDS cohort' (stage III NVRD after PDS) and c) a larger 'simulated-broader cohort' (both cohorts $\mathrm{a}$ and $\mathrm{b}$ plus the additional stage III nonevaluable debulking status cases).

Methodology A retrospective observational study was conducted to examine characteristics and long-term outcomes for patients diagnosed with advanced OC within the Edinburgh Cancer Centre between 1 January 2000 and 31 December 2015. Patients were followed until their last patient record or last data cut (January 2019) and were chosen to match the three defined populations. Main outcomes were OS and PFS, the latter defined as time from diagnosis to first progression as defined by radiology, tumour marker (CA125) or the treating physician where other investigations were not evaluable.

Results Baseline patient characteristics in the simulated-PRIMA $(\mathrm{n}=472)$, simulated-stage III NVRD after PDS $(\mathrm{n}=69)$ and simulated-broader $(n=569)$ cohorts matched the PRIMA trial in most categories. PFS and OS for simulated cohorts are shown in the table 1 . When compared with the simulatedPRIMA cohort, the simulated-stage III NVRD after PDS

Abstract 367 Table 1
\begin{tabular}{|l|c|c|}
\hline Cohort & Median PFS, yr & Median os, $\mathbf{y r}$ \\
\hline Simulated-PRIMA ( $n=472)$ & 1.20 & 2.71 \\
\hline Simulated-stage III NVRD after PDS ( $n=69)$ & 2.45 & 6.84 \\
\hline Simulated-broader ( $n=569$ ) & 1.26 & 3.07 \\
\hline NVRD, no visible residual disease; OS, overall survival; PDS, primary debulking surgery; \\
PFS, progression-free survival.
\end{tabular}

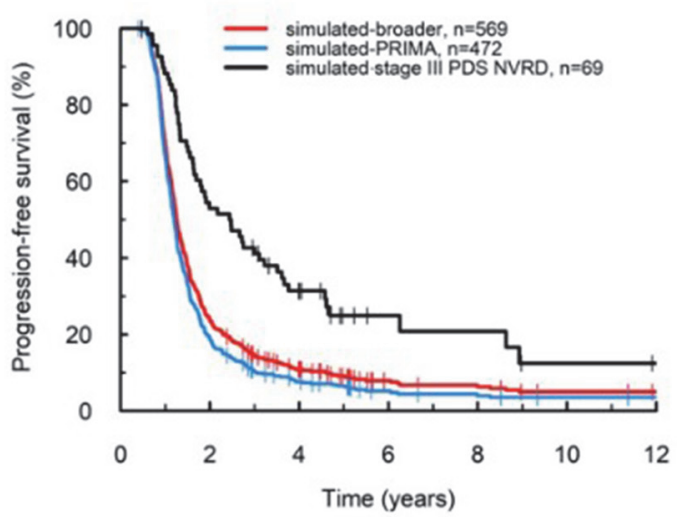


cohort had significantly longer PFS (hazard ratio $[\mathrm{HR}]=0.43$, $95 \%$ CI $0.32-0.58, \mathrm{P}<0.0001)$ and significantly longer OS $(\mathrm{HR}=0.43,95 \%$ CI $0.32-0.60, \mathrm{P}<0.0001)$. Furthermore, the simulated-broader cohort demonstrated a survival curve above the simulated-PRIMA curve. Within the 2010-2015 diagnosis (contemporary) stage III cohort $(n=169), 57.4 \%$ had IDS and 42.6\% had PDS, of whom $23.1 \%$ had PDS VRD, $17.2 \%$ had PDS NVRD, and 2.4\% were PDS not evaluable for residual disease.

Conclusion The simulated-broader cohort showed longer duration of OS and PFS outcomes as the survival curves lie above the simulated-PRIMA cohort. This difference is driven by the better prognosis for patients with stage III NVRD after PDS population; this population accounted for approximately $17 \%$ of the contemporary patient cohort at this UK centre.

Disclosures This study was funded by GlaxoSmithKline.

Clinical Trial Registration: N/A

Dr. Hollis reports institutional grants and personal fees from GlaxoSmithKline.

Dr. Gourley reports personal fees from Roche, Clovis, Tesaro, Foundation One, Nucana, Aprea, Novartis, Chugai, and MSD; institutional grants from Clovis, Tesaro, Nucana, and Novartis.

Drs. Kiss, Roebuck, Heffernan and Starkie-Camejo are employees of GlaxoSmithKline.

\section{SYSTEMATIC LITERATURE REVIEW OF EFFICACY AND SAFETY OF FIRST-LINE MAINTENANCE THERAPY TRIALS IN ADVANCED OVARIAN CANCER}

${ }^{1}$ Holly Guy, ${ }^{2}$ Karin Travers, ${ }^{2}$ Carol Hawkes, ${ }^{1}$ Lydia Walder, ${ }^{2}$ Izabela Malinowska,

${ }^{2}$ Divya Gupta. ${ }^{1}$ Fiecon; ${ }^{2}$ Glaxosmithkline

\subsection{6/ijgc-2020-ESG0.127}

Introduction/Background Maintenance therapies have changed the treatment landscape in ovarian cancer (OC) in recent years. Here we sought to review safety and efficacy outcomes in clinical trials of first-line maintenance therapies.

Methodology A systematic literature review (SLR) was performed on 27 February 2020 to identify clinical outcomes associated with first-line maintenance therapies and maintenance therapies initiated alongside first-line chemotherapy followed by a maintenance phase for advanced OC. Randomised controlled trials (RCTs), non-RCTs and observational studies were eligible. Selection criteria following the PICOS (population, interventions, comparators, outcomes and study type) principle were specified. Outcomes of interest were progression-free survival (PFS), overall survival (OS), and treatment emergent adverse events (TEAEs). Selected studies were then extracted by one reviewer and assessed for quality by a second reviewer. Disagreements were resolved by a third reviewer when required.

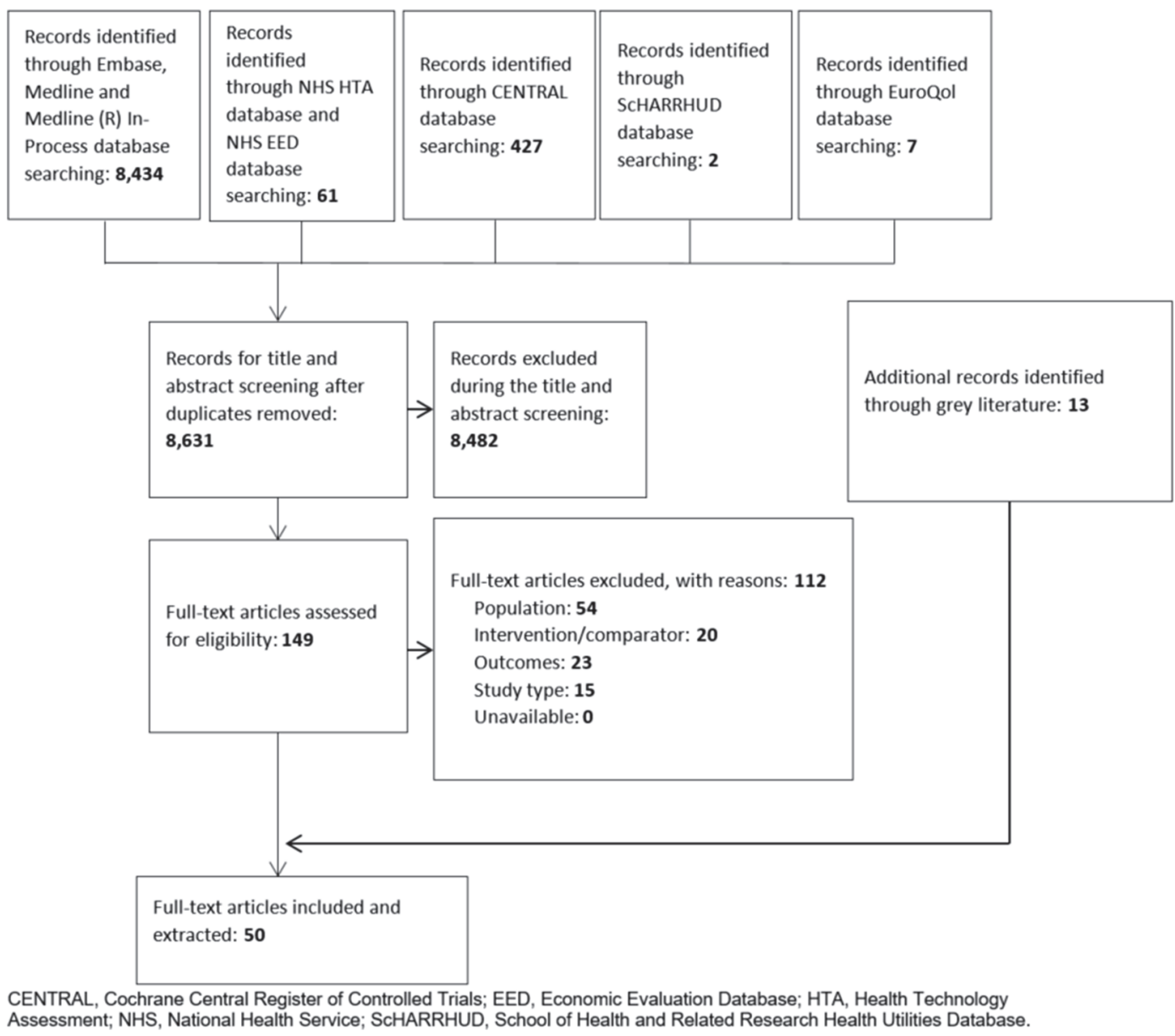

Abstract 373 Figure 1 PRISMA diagram 